\title{
A characterization of covering equivalence
}

\author{
by \\ HaO PAn (Shanghai) and ZhI-WeI Sun (Nanjing)
}

1. Introduction. For $n \in \mathbb{Z}^{+}=\{1,2,3, \ldots\}$ and $a \in\{0, \ldots, n-1\}$, we write $a(n)$ to denote the residue class $\{x \in \mathbb{Z}: x \equiv a(\bmod n)\}$. For a finite system

$$
A=\left\{a_{s}\left(n_{s}\right)\right\}_{s=1}^{k} \quad\left(0 \leq a_{s}<n_{s}\right)
$$

of residue classes, the $n_{1}, \ldots, n_{k}$ are called its moduli, and its covering function $w_{A}: \mathbb{Z} \rightarrow \mathbb{N}=\{0,1, \ldots\}$ is given by

$$
w_{A}(x)=\left|\left\{1 \leq s \leq k: x \in a_{s}\left(n_{s}\right)\right\}\right| .
$$

(The covering function $w_{\emptyset}$ of an empty system is regarded as the zero function.) The periodic function $w_{A}(x)$ has many surprising properties (cf. [S03a], [S04] and [S05a]).

Let $m$ be a positive integer. If $w_{A}(x)=m$ for all $x \in \mathbb{Z}$, then (1.1) is said to be an exact $m$-cover of $\mathbb{Z}$ as in [S95] and [S96]. Recently Z. W. Sun (cf. [S04] and [S05b]) showed that (1.1) forms an exact $m$-cover of $\mathbb{Z}$ if it covers $\left|S\left(n_{1}, \ldots, n_{k}\right)\right|$ consecutive integers exactly $m$ times, where

$$
S\left(n_{1}, \ldots, n_{k}\right)=\left\{r / n_{s}: r=0, \ldots, n_{s}-1 ; s=1, \ldots, k\right\} .
$$

For problems and results on covers of $\mathbb{Z}$ by residue classes, the reader is referred to [FFKPY], [G04] and [S03b].

For two finite systems $A=\left\{a_{s}\left(n_{s}\right)\right\}_{s=1}^{k}$ and $B=\left\{b_{t}\left(m_{t}\right)\right\}_{t=1}^{l}$, Sun [S89] called $A$ and $B$ covering equivalent (written $A \sim B$ ) if they have the same covering function (i.e., $w_{A}=w_{B}$ ). Thus (1.1) is an exact $m$-cover of $\mathbb{Z}$ if and only if (1.1) is covering equivalent to the system consisting of $m$ copies of $0(1)$.

In [S01] and [S02] Sun characterized the covering equivalence by various systems of equalities. In this paper we present a simple characterization involving roots of unity. Namely, we have the following result.

2000 Mathematics Subject Classification: Primary 11B25; Secondary 11A07, $11 B 75$.

Key words and phrases: covering equivalence, Graham-O'Bryant conjecture.

The second author is responsible for communications, and supported by the National Science Fund (Grant No. 10425103) for Distinguished Young Scholars in China. 
TheOREM 1.1. Let $A=\left\{a_{s}\left(n_{s}\right)\right\}_{s=1}^{k}\left(0 \leq a_{s}<n_{s}\right)$ and $B=\left\{b_{t}\left(m_{t}\right)\right\}_{t=1}^{l}$ $\left(0 \leq b_{t}<m_{t}\right)$ be two finite systems of residue classes. Let $p$ be a prime greater than $\left|S\left(n_{1}, \ldots, n_{k}, m_{1}, \ldots, m_{l}\right)\right|$, and let $\zeta_{p}$ be a primitive pth root of unity. Then $A$ and $B$ are covering equivalent if and only if

$$
\sum_{s=1}^{k} \frac{\zeta_{p}^{a_{s}}}{1-\zeta_{p}^{n_{s}}}=\sum_{t=1}^{l} \frac{\zeta_{p}^{b_{t}}}{1-\zeta_{p}^{m_{t}}} .
$$

COROLlary 1.1. (1.1) forms an exact $m$-cover of $\mathbb{Z}$ if and only if

$$
\sum_{s=1}^{k} \frac{e^{2 \pi i a_{s} / p}}{1-e^{2 \pi i n_{s} / p}}=\frac{m}{1-e^{2 \pi i / p}},
$$

where $p$ is any fixed prime greater than $\left|S\left(n_{1}, \ldots, n_{k}\right)\right|$.

Proof. Simply apply Theorem 1.1 with $B$ consisting of $m$ copies of $0(1)$.

REMARK 1.1. In 1975 Š. Znám [Z75a] used the transcendence of $e$ to prove that (1.1) is a disjoint cover (i.e., exact 1 -cover) of $\mathbb{Z}$ if and only if

$$
\sum_{s=1}^{k} \frac{e^{a_{s}}}{1-e^{n_{s}}}=\frac{1}{1-e} .
$$

Corollary 1.2. Suppose that for a nonempty system (1.1) we have

$$
\sum_{s=1}^{k} \frac{e^{2 \pi i a_{s} / p}}{1-e^{2 \pi i n_{s} / p}}=0
$$

where $p$ is a prime. Then

$$
n_{1}+\cdots+n_{k}-k+1 \geq\left|S\left(n_{1}, \ldots, n_{k}\right)\right| \geq p .
$$

Proof. Clearly $\left|S\left(n_{1}, \ldots, n_{k}\right)\right| \leq n_{1}+\cdots+n_{k}-k+1$. Since $A \not \emptyset$, applying Theorem 1.1 with $B=\emptyset$ we find that $\left|S\left(n_{1}, \ldots, n_{k}\right)\right|$ cannot be smaller than $p$.

Corollary 1.2 partially confirms the following conjecture arising from the study of Fraenkel's conjecture on disjoint covers of $\mathbb{N}$ by Beatty sequences.

Graham-O'Bryant Conjecture $([\mathrm{GO}])$. Let $n_{1}, \ldots, n_{k}$ be distinct positive integers less than and relatively prime to $q \in \mathbb{Z}^{+}$. If $a_{1}, \ldots, a_{k} \in \mathbb{Z}$ and

$$
\sum_{s=1}^{k} \frac{e^{2 \pi i a_{s} / q}}{1-e^{2 \pi i n_{s} / q}}=0
$$

then we must have $\sum_{s=1}^{k} n_{s} \geq q$.

The following example shows that we cannot replace the prime $p$ in Corollary 1.2 or Theorem 1.1 by a composite number. 
EXAMPle 1.1. Let $q>1$ be an integer and let $p$ be a prime divisor of $q$. Then, for any $n=1, \ldots, q-1$, we have

$$
\sum_{s=0}^{p-1} \frac{e^{2 \pi i(s q / p) / q}}{1-e^{2 \pi i n / q}}=\frac{\sum_{s=0}^{p-1} e^{2 \pi i s / p}}{1-e^{2 \pi i n / q}}=0
$$

but $|S(n, \ldots, n)|=n<q$. Thus the conditions $0 \leq a_{s}<n_{s}(s=1, \ldots, k)$ in Corollary 1.2 cannot be cancelled. If $q$ is composite, then there are $q / p-1>0$ integers in the interval $((p-1) q / p, q-1]$. So we cannot substitute a composite number for the prime $p$ in Corollary 1.2.

Corollary 1.3. Let $A=\left\{a_{s}\left(n_{s}\right)\right\}_{s=1}^{k}\left(0 \leq a_{s}<n_{s}\right)$ and $B=$ $\left\{b_{t}\left(m_{t}\right)\right\}_{t=1}^{l}\left(0 \leq b_{t}<m_{t}\right)$ both have distinct moduli. Let $p$ be a prime greater than $\left|S\left(n_{1}, \ldots, n_{k}, m_{1}, \ldots, m_{l}\right)\right|$, and let $\zeta_{p}$ be a primitive pth root of unity. Then $A$ and $B$ are identical if and only if (1.4) holds.

Proof. By a result of Znám [Z75b], $A$ and $B$ are identical if they have the same covering function. Combining this with Theorem 1.1 we immediately get the desired result.

Observe that $A=\left\{a_{s}\left(n_{s}\right)\right\}_{s=1}^{k}$ and $B=\left\{b_{t}\left(m_{t}\right)\right\}_{t=1}^{l}$ are covering equivalent if and only if

$$
\sum_{\substack{s=1 \\ x \in a_{s}\left(n_{s}\right)}}^{k} 1+\sum_{\substack{t=1 \\ x \in b_{t}\left(m_{t}\right)}}^{l}(-1)=0 \quad \text { for every } x \in \mathbb{Z} .
$$

Thus Theorem 1.1 has the following equivalent form which will be proved in the next section.

TheOREM 1.2. Let $\mathcal{A}=\left\{\left\langle\lambda_{s}, a_{s}, n_{s}\right\rangle\right\}_{s=1}^{k}$ where $\lambda_{s}, a_{s}, n_{s} \in \mathbb{Z}$ and $0 \leq$ $a_{s}<n_{s}$. Let $p>\left|S\left(n_{1}, \ldots, n_{k}\right)\right|$ be a prime, and let $\zeta_{p}$ be any primitive $p$ th root of unity. Then $\mathcal{A} \sim \emptyset$ (i.e., $w_{\mathcal{A}}(x)=\sum_{1 \leq s \leq k, x \in a_{s}\left(n_{s}\right)} \lambda_{s}=0$ for all $x \in \mathbb{Z})$ if and only if

$$
\sum_{s=1}^{k} \lambda_{s} \frac{\zeta_{p}^{a_{s}}}{1-\zeta_{p}^{n_{s}}}=0 .
$$

2. Proof of Theorem 1.2. Let $S=S\left(n_{1}, \ldots, n_{k}\right)$. As $p>|S| \geq$ $\max \left\{n_{1}, \ldots, n_{k}\right\}$, there is a common multiple $N \in \mathbb{Z}^{+}$of the moduli $n_{1}, \ldots, n_{k}$ such that $N \equiv 1(\bmod p)$. Just as in [S05a], we have

$$
\sum_{r=0}^{N-1} w_{\mathcal{A}}(r) z^{r}=\sum_{r=0}^{N-1} \sum_{\substack{1 \leq s \leq k \\ n_{s} \mid a_{s}-r}} \lambda_{s} z^{r}=\sum_{s=1}^{k} \lambda_{s} \sum_{\substack{0 \leq r<N \\ r \in a_{s}\left(n_{s}\right)}} z^{r}
$$




$$
\begin{aligned}
& =\sum_{s=1}^{k} \lambda_{s} z^{a_{s}} \sum_{0 \leq q<N / n_{s}}\left(z^{n_{s}}\right)^{q}
\end{aligned}
$$

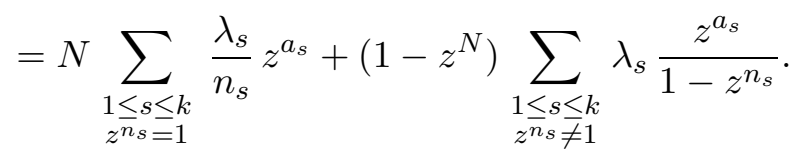

Thus

$$
\sum_{r=0}^{N-1} w_{\mathcal{A}}(r) \zeta_{p}^{r}=\left(1-\zeta_{p}^{N}\right) \sum_{s=1}^{k} \lambda_{s} \frac{\zeta_{p}^{a_{s}}}{1-\zeta_{p}^{n_{s}}}
$$

It follows that

$$
\sum_{s=1}^{k} \lambda_{s} \frac{\zeta_{p}^{a_{s}}}{1-\zeta_{p}^{n_{s}}}=0 \Leftrightarrow \sum_{l=0}^{p-1} c_{l} \zeta_{p}^{l}=0
$$

where

$$
c_{l}=\sum_{\substack{x=0 \\ x \in l(p)}}^{N-1} w_{\mathcal{A}}(x) \in \mathbb{Z} .
$$

If $w_{\mathcal{A}}(x)=0$ for all $x \in \mathbb{Z}$, then (1.7) holds by the above.

Below we assume (1.7). Then $\sum_{l=0}^{p-1} c_{l} \zeta_{p}^{l}=\sum_{r=0}^{N-1} w_{\mathcal{A}}(r) \zeta_{p}^{r}=0$. In the case $N=1$, it follows that $w_{\mathcal{A}}(x)=w_{\mathcal{A}}(0)=0$ for all $x \in \mathbb{Z}$. Now suppose $N>1$. Clearly $N>p$ as $N \equiv 1(\bmod p)$. Since $1+x+\cdots+x^{p-1}=$ $\left(x^{p}-1\right) /(x-1)$ is the minimal polynomial of $\zeta_{p}$ over the field of rational numbers, we must have $c_{0}=c_{1}=\cdots=c_{p-1}$. (See also M. Newman [N71].) Observe that if $x \in \mathbb{Z}$ then

$$
w_{\mathcal{A}}(x)=\sum_{s=1}^{k} \frac{\lambda_{s}}{n_{s}} \sum_{r=0}^{n_{s}-1} e^{2 \pi i \frac{a_{s}-x}{n_{s}} r}=\sum_{\alpha \in S} e^{-2 \pi i \alpha x} \sum_{\substack{s=1 \\ \alpha n_{s} \in \mathbb{Z}}}^{k} \frac{\lambda_{s}}{n_{s}} e^{2 \pi i \alpha a_{s}} .
$$

(This trick appeared in [S91] and [S04].) Since $|S|<p$, for each $l=0, \ldots,|S|$ we have

$$
\begin{aligned}
c_{l} & =\sum_{\substack{x=0 \\
x \in l(p)}}^{N-1} w_{\mathcal{A}}(x)=\sum_{\alpha \in S} \sum_{\substack{s=1 \\
\alpha n_{s} \in \mathbb{Z}}}^{k} \frac{\lambda_{s}}{n_{s}} e^{2 \pi i \alpha a_{s}} \sum_{\substack{x=0 \\
x \in l(p)}}^{N-1} e^{-2 \pi i \alpha x} \\
& =\sum_{\alpha \in S} e^{-2 \pi i \alpha l} \sum_{\substack{s=1 \\
\alpha n_{s} \in \mathbb{Z}}}^{k} \frac{\lambda_{s}}{n_{s}} e^{2 \pi i \alpha a_{s}} \sum_{j=0}^{\lfloor(N-1-l) / p\rfloor} e^{-2 \pi i \alpha p j},
\end{aligned}
$$

where $\lfloor\cdot\rfloor$ is the greatest integer function. If $l \in\{1, \ldots,|S|\}$ then

$$
\left\lfloor\frac{N-1-l}{p}\right\rfloor=\frac{N-1}{p}+\left\lfloor\frac{-l}{p}\right\rfloor=\frac{N-1}{p}-1 ;
$$


if $\alpha \in S \backslash\{0\}$ then

$$
C(\alpha):=\sum_{j=0}^{(N-1) / p-1} e^{-2 \pi i \alpha p j}=\frac{1-\left(e^{-2 \pi i \alpha p}\right)^{(N-1) / p}}{1-e^{-2 \pi i \alpha p}}=\frac{1-e^{2 \pi i \alpha}}{1-e^{-2 \pi i \alpha p}} \neq 0 .
$$

Let $c=c_{0}=\cdots=c_{p-1}$. By the above,

$$
\sum_{\alpha \in S} e^{-2 \pi i \alpha j} f(\alpha)=c
$$

for every $j=0, \ldots,|S|-1$, where

$$
f(0)=\frac{N-1}{p} \sum_{s=1}^{k} \frac{\lambda_{s}}{n_{s}}
$$

and

$$
f(\alpha)=e^{-2 \pi i \alpha} C(\alpha) \sum_{\substack{s=1 \\ \alpha n_{s} \in \mathbb{Z}}}^{k} \frac{\lambda_{s}}{n_{s}} e^{2 \pi i \alpha a_{s}} \quad \text { for } \alpha \in S \backslash\{0\} .
$$

Let $\alpha_{0}=0, \alpha_{1}, \ldots, \alpha_{|S|-1}$ be all the distinct elements of $S$. Now that

$$
\sum_{t=0}^{|S|-1} e^{-2 \pi i \alpha_{t} j} f\left(\alpha_{t}\right)=c \quad \text { for each } j=0, \ldots,|S|-1,
$$

by Cramer's rule $D_{t}=D f\left(\alpha_{t}\right)$ vanishes for every $t=1, \ldots,|S|-1$, where $D=\operatorname{det}\left(\left(e^{-2 \pi i \alpha_{t}}\right)^{j}\right)_{0 \leq j, t<|S|}$ is of Vandermonde's type and hence nonzero. Therefore

$$
\sum_{\substack{s=1 \\ \alpha n_{s} \in \mathbb{Z}}}^{k} \frac{\lambda_{s}}{n_{s}} e^{2 \pi i \alpha a_{s}}=0 \quad \text { for all } \alpha \in S \backslash\{0\}
$$

and hence $w_{\mathcal{A}}(x)=\sum_{s=1}^{k} \lambda_{s} / n_{s}$ for all $x \in \mathbb{Z}$ by $(2.2)$. It follows that

$$
0=\sum_{r=0}^{N-1} w_{\mathcal{A}}(r) \zeta_{p}^{r}=\sum_{s=1}^{k} \frac{\lambda_{s}}{n_{s}}\left(1+\zeta_{p}+\zeta_{p}^{2}+\cdots+\zeta_{p}^{N-1}\right)=\sum_{s=1}^{k} \frac{\lambda_{s}}{n_{s}} \cdot \frac{1-\zeta_{p}^{N}}{1-\zeta_{p}} .
$$

So $\sum_{s=1}^{k} \lambda_{s} / n_{s}=0$ and hence $\mathcal{A} \sim \emptyset$. We are done.

\section{References}

[FFKPY] M. Filaseta, K. Ford, S. Konyagin, C. Pomerance and G. Yu, Sieving by large integers and covering systems of congruences, J. Amer. Math. Soc. 20 (2007), 495-517.

[GO] R. L. Graham and K. O'Bryant, A discrete Fourier kernel and Fraenkel's tiling conjecture, Acta Arith. 118 (2005), 283-304. 
[G04] R. K. Guy, Unsolved Problems in Number Theory, 3rd ed., Springer, New York, 2004.

[N71] M. Newman, Roots of unity and covering sets, Math. Ann. 191 (1971), 279282.

[S89] Z. W. Sun, Systems of congruences with multipliers, Nanjing Univ. J. Math. Biquarterly 6 (1989), no. 1, 124-133.

[S91] - On a generalization of a conjecture of Erdös, Nanjing Univ. J. Natur. Sci. 27 (1991), no. 1, 8-15.

[S95] - Covering the integers by arithmetic sequences, Acta Arith. 72 (1995), 109129.

[S96] - Covering the integers by arithmetic sequences II, Trans. Amer. Math. Soc. 348 (1996), 4279-4320.

[S01] - Algebraic approaches to periodic arithmetical maps, J. Algebra 240 (2001), 723-743.

[S02] - On covering equivalence, in: Analytic Number Theory (Beijing/Kyoto, 1999), Dev. Math. 6, Kluwer, Dordrecht, 2002, 277-302.

[S03a] - On the function $w(x)=\left|\left\{1 \leq s \leq k: x \equiv a_{s}\left(\bmod n_{s}\right)\right\}\right|$, Combinatorica 23 (2003), 681-691.

[S03b] - Unification of zero-sum problems, subset sums and covers of $\mathbb{Z}$, Electron. Res. Announc. Amer. Math. Soc. 9 (2003), 51-60.

[S04] -, Arithmetic properties of periodic maps, Math. Res. Lett. 11 (2004), 187196.

[S05a] - On the range of a covering function, J. Number Theory 111 (2005), 190196.

[S05b] -, A local-global theorem on periodic maps, J. Algebra 293 (2005), 506-512.

[Z75a] Š. Znám, A simple characterization of disjoint covering systems, Discrete Math. 12 (1975), 89-91.

[Z75b] - On properties of systems of arithmetic sequences, Acta Arith. 26 (1975), 279-283.

Department of Mathematics

Shanghai Jiaotong University

Shanghai 200240

People's Republic of China

E-mail: haopan79@yahoo.com.cn
Department of Mathematics Nanjing University Nanjing 210093

People's Republic of China E-mail: zwsun@nju.edu.cn 